\title{
CONCEPCIONES HISTÓRICAS ASOCIADAS AL CONCEPTO DE LÍMITE E IMPLICACIONES DIDÁCTICAS
}

\author{
Ana Cecilia Medina M. \\ Estudiante del Programa de Maestría en Docencia de la Matemática. \\ Universidad Pedagógica Nacional
}

\begin{abstract}
In this article, a synthesis of an approximate fo the study of the different conceptions relative to the concept of "limit, identified throughout its historical evolution, and of the role that this epistemological study plays in an investigation, developed under the framework of the comprehension of the mathematical concepts, is presented.
\end{abstract}

\section{RESUMEN}

En este artículo se presenta una síntesis de un estudio aproximativo de las concepciones históricas relativas al concepto de "límite, identificadas en su génesis y evolución, y el papel que desempeña este estudio epistemológico en un trabajo de investigación desarrollado en el marco de la comprensión de los conceptos matemáticos.

\section{INTRODUCCIÓN}

El trabajo que aquí se presenta posee una doble intencionalidad: primero, caracterizar las principales concepciones identificadas en la evolución histórica de la noción de límite y segundo, generar reflexión acerca de la enseñanza y aprendizaje del cálculo. Este estudio hace parte del trabajo de tesis titulado "Imágenes del concepto de límite en estudiantes universitarios", el cual se encuadra en la línea de investigación "Una aproximación epistemológica, didáctica y cognitiva al cálculo", propuesta por la Maestría en Docencia de la Matemática de Fa Universidad Pedagógica Nacional, que se orienta a "estudiar los fenómenos de la enseñanza, aprendizaje y comunicación de las nociones básicas y conceptos del cálculo" (García, G., Serrano, O., Díaz, H., 1999). Para abordar el contenido de esta presentación, se comienza con algunas razones que explican la importancia del estudio epistemológico de la noción de limite, luego los referentes teóricos que apoyan el modelo de Análisis para después presentar. a manera de síntesis. la descripción de cada categoría de concepciones históricas relativas a la noción de limite, los problemas conceptuales, las dificultades y obstáculos epistemológicos asociados a cada una de estas concepciones y concluir con algunas reflexiones e interrogantes sobre sus implicaciones didácticas. 


\section{ALGUNOS ASPECTOS DEL ESTUDIO EPISTEMOLÓGICO DE LA NOCIÓN DE LÍMITE}

\section{1 ¿Por qué el concepto de límite?}

El concepto de "límite" ocupa una posición central en el campo conceptual del cálculo y su complejidad resulta ser fuente de dificultades tanto en la enseñanza como en el aprendizaje. Primero por su carácter estructural que lo constituye el eje central y concepto básico sobre el cual se construye la estructura del Cálculo diferencial e integral y otros conceptos de otras ramas de la matemática; también por su carácter instrumental como herramienta para la solución de problemas tanto al interior de las matemáticas como de ciencias aplicadas como la Física, la Ingeniería y finalmente, como objeto matemático que se gesta en diferentes con-textos: geométrico, aritmético, métrico, topológico y asociado a otros objetos matemáticos

\subsection{Tratamiento didáctico tradicional}

Una de las intencionalidades del educador matemático es la búsqueda de la "comprensión" como construcción y apropiación de significados relativos a elementos constitutivos (Sierpinska, 1998), de los conceptos matemáticos por parte de los estudiantes, lo cual genera interrogantes acerca de su "actividad matemática", como: ¿Qué concepciones o imágenes del concepto se forman? ¿Estas concepciones los aproximan al concepto? ¿Cómo aprenden el concepto?

Estos interrogantes cobran mayor importancia cuando se privilegia el acercamiento al concepto de límite por medio de la definición formal $\varepsilon-\delta$. Al respecto, investigaciones en Educación Matemática han evidenciado que "la definición de un concepto no garantiza la comprensión del concepto" (Tall y Vinner, 1981), ya que la definición formal encapsula las situaciones y procesos que le dan sentido al concepto, ocultando su significación y complejidad.

\section{3 ¿Por qué el estudio de las concepciones históricas?}

Como el objetivo principal de este trabajo de investigación es estudiar las concepciones que manifiestan los estudiantes acerca de la noción de límite, se debe tener como punto de partida los significados atribuidos al concepto, los cuales no se construyen consultando los libros de texto de Cálculo más elevados, porque allí por una parte se encuentra el conocimiento matemático terminado y pulido con su apariencia perfecta inequívoca y rigurosa, ocultando las dificultades y obstáculos que hicieron evolucionar la noción hasta llegar a institucionalizarse como concepto matemático; y por otra, los textos portan concepciones de la noosfera, es decir, del sistema político, económico y educativo de un país (Chevallard, 1991), de los autores y otras concepciones que se inducen por la inserción de objetos didácticos para convertir el conocimiento en saber escolar. A este conjunto de adaptaciones que experimenta un conocimiento desde su estado de saber científico (aceptado por la comunidad científica) hasta convertirse en saber escolar y en un sentido más amplio hasta convertirse en saber del alumno, proceso en que interviene también las adaptaciones del maestro y la relación didáctica "es lo que Chevallard (1991) ha denominado Transposición Didáctica.

Por lo tanto, para acercarnos a la configuración de los elementos constitutivos de la significación del concepto la mejor alternativa es ir a fuentes que muestran la forma de 
producción del conocimiento como la Historia de las Matemáticas e Investigaciones en Educación Matemática.

\subsection{El modelo de análisis}

Este estudio aproximativo de la evolución de la noción de límite se ha apoyado en las teorías sobre concepciones de Vergnaud (1982, citado por Ruiz, 1993), problemas conceptuales de Toulmin (1977. citado por Delgado, 1998), dificultades y obstáculos epistemológicos de Brousseau (citado por Ruiz, 1993) y El Bouazzaoui (1988) y el estatus de un concepto matemático de Chevallard (1991) y Sfard (1991).

Para el estudio de las concepciones históricas de la noción de límite se adoptó la definición de concepción de Vergnaud, quién parte de la definición de un concepto matemático:

"Un concepto matemático está determinado por una terna $(S, I, S)$ en donde $S$ : es el conjunto de situaciones que dan sentido al concepto, I: el conjunto de invariantes que constituyen el concepto y s: el conjunto de representaciones simbólicas usadas para presentar el concepto, sus propiedades y las situaciones a las que se refiere."

Análogamente una concepción estaría formada por esta misma terna, pero considerándola en un momento dado de la evolución del concepto"(Vergnaud, $1982 b$, citado por Ruiz, 1993)

Esta definición proporciona elementos que permiten hacer un seguimiento de la evolución de la noción para diferenciar y caracterizar las diferentes etapas en las que los matemáticos la manipularon de acuerdo con las preocupaciones de cada época.

El análisis de la evolución de las concepciones se apoyó en la Teoría de dificultades y obstáculos epistemológicos de Brousseau y El Bouazzaoui (1988) quienes consideran que la evolución de una noción matemática para convertirse en concepto hace que la elaboración del mismo, sea un proceso en el que aparecen dificultades y obstáculos. Cada concepción histórica permite la resolución de un conjunto de situaciones problema, llamado dominio de aplicación de la concepción. Una "dificultad" aparece cuando un problema nuevo se resuelve reorganizando la teoría de la concepción que se dispone, pero si para la solución se requiere de un cambio importante o radical de punto de vista, o sea se requiere una nueva concepción, se dice que un "obstáculo" ha sido superado.

A estos obstáculos presentados en el desarrollo histórico del conocimiento se les ha denominado "Obstáculos epistemológicos", término introducido por Bachelard para afirmar que:

"El conocimiento científico no se desarrolla en un proceso continuo, sino que resulta del rechazo de formas previas de conocimiento que se constituyen en obstáculos epistemológicos" (Bachelard, 1993).

De otra parte, el estudio filogenético de los conceptos revelan que las nociones sufren un proceso lento de institucionalización, en el cual pasan de ser procesos de uso, a ser instrumentos de la matemática y finalmente a objetos de estudio (Chevallard, 1998), en el que intervienen factores internos de la Matemática como disciplina científica y externos de los entornes social, cultural y científico de la época. Punto de vista que se complementa con el expuesto por Sfard (1991) quien sostiene que muchas nociones matemáticas 
pueden concebirse de dos formas: operacionalmente o como proceso y estructuralmente, como objete de una forma estática; y la posibilidad de manejar con eficacia estas dos dimensiones requiere proceso cognitivos especiales como la reificación que convierte un proceso en un objeto abstracto.

\section{CONCEPCIONES HISTÓRICAS RELATIVAS AL CONCEPTO DE LÍMITE}

Hacer un seguimiento a la evolución histórica de la noción de límite, implica hacer un recorrido histórico a las Matemáticas, encontrando que esta noción no se desarrollé en forma independiente y autónoma sino que hace parte de una red

o entramado que se obtiene por medio de la interacción e interdependencia con otras nociones vecinas del cálculo; variable, función, función continua, infinito, infinitesimal, número, número real, continuo numérico.

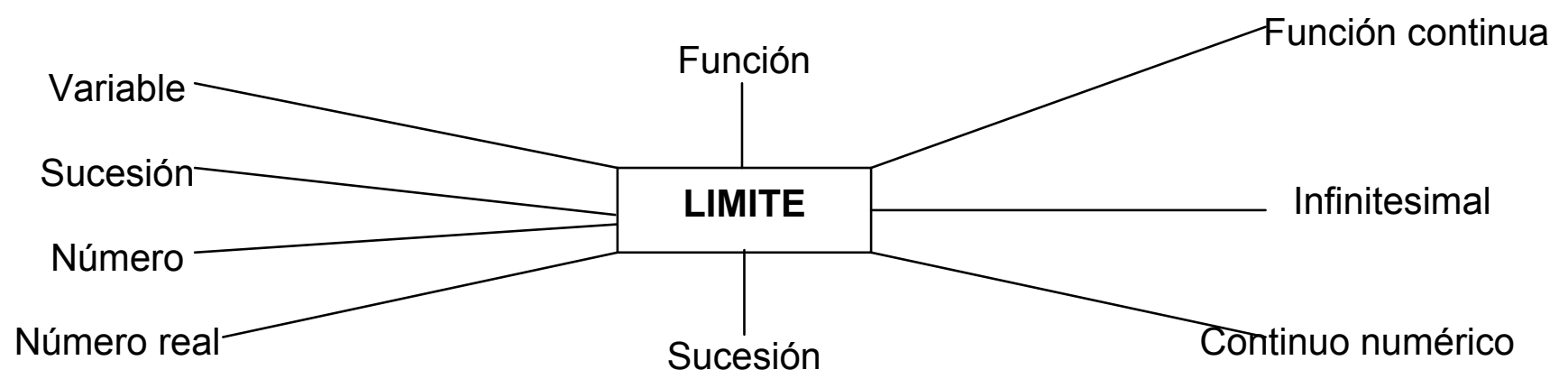

Para facilitar el análisis se tuvo en cuenta el contexto matemático, en el que se fue desarrollando la noción a excepción de las concepciones de Newton y Leibniz, las cuales se analizan por separado, debido a los matices particulares que poseen. Es importante aclarar que estas categorías de concepciones no son independientes, sino que permanentemente se interrelacionan y afectan mutuamente. En cada una se ha pretendido agrupar el pensamiento de la comunidad matemática de la época y se elige un matemático como representante aunque también en una misma época coexisten posturas divergentes.

\subsection{Concepción geométrica}

Está relacionada con situaciones ligadas al contexto geométrico y tienen su origen en el origen de las matemáticas cuyo énfasis fue geométrico. Se han considerado tres categorías de concepciones correspondientes a tres épocas:

- Antigüedad Griega - época clásica

(S. VI a. C. - S. III a. C.) 


\section{CONCEPCIÓN GEOMÉTRICA \\ RIGUROSA - EUDOXO - EUCLIDES}

El paso al limite no es una operación matemática, sino que está oculta en el método de exhaución, para probar ciertas relaciones entre magnitudes. (Síerpinska, 1987 pág 32).

RIGUROSA: Método de exhaución de Eudoxo. Se privilegian las demostraciones por doble reducción al absurdo, para probar relaciones entre magnitudes geométricas, deducidas intuitivamente, más que el hallazgo de resultados.

- Época Greco - Alejandrina

(S. III a.C. - S II)

\section{CONCEPCIÓN GEOMÉTRICA HEURÍSTICA - RIGUROSA - ARQUÍMEDES}

El paso al limite está implícito en el método heurístico de aproximaciones sucesivas" que conduce a hallazgos gracias a la intuición geométrica y conocimiento sobre mecánica y que luego demuestra por reducción al absurdo.

HEURISTICA - RIGUROSA: Con su "método mecánico" (oculto) y de aproximaciones sucesivas" encuentra resultados que luego prueba por el método de exhaución.

En la época griega se presentan situaciones que dan oportunidad a las primeras manifestaciones intuitivas de la idea de límite. Ellas tienen que ver con el encuentro de procesos geométricos infinitos que surgen de las paradojas de Zenón, el descubrimiento de los inconmensurables o irracionales y la comparación de áreas y volúmenes de figuras curvilíneas por aproximación de figuras rectilíneas. Por ejemplo el problema de calcular el área del círculo proporcionó una oportunidad para desarrollar herramientas muy similares al concepto de límite. (Oornu, 1991, p. 159)

- Renacimiento (S. XV - S. XVII)

\section{CONCEPCIÓN GEOMETRICA HEURÍSTICA DE APROXIMACIÓN FINITA - CAVALIERI - KEPLER}

El paso al limite no es una operación matemática, pero está implícito en un método heuristico que permite la búsqueda de lo que no conocemos más que aproximaciones.

HEURISTICA: Se acude a la intuición geométrica, al libre uso del infinito y extensos cálculos numéricos, para hallar resultados cuantitativos. 
A finales del siglo XVI y comienzos del siglo XVII, los matemáticos del renacimiento, basados en el método de Arquímedes, pero tratando de evadir la rigurosidad del método de exhaución y aprovechando la inserción del infinito en los razonamientos matemáticas por algunos filósofos escolásticos, surgen nuevos métodos para resolver problemas de áreas de figuras curvilíneas. Por ejemplo tanto Stevin (1548 - 1620) como Luca Valerio en 1604 se aproximaron a la idea de límite aunque en lo geométrico al indicar la condición necesaria para la existencia de un límite - a saber, "que la diferencia entre determinadas áreas puede hacerse menor que un área específica". (Boyer, 1959, p. 105, 106) 48

Cavalieri (1598- 1647) propone el "método de los indivisibles "para comparar áreas y volúmenes, método que ocupa un lugar intermedio entre las rigurosas concepciones de Arquímedes y los nuevos métodos infinitesimales de Newton y Leibniz Y Kepler (1571 — 1630) inicia los planteamientos del infinitamente pequeño, para el cálculo de áreas, superficies barridas en el movimiento planetario, volúmenes, estudio de curvas representando trayectorias de movimientos.

\section{Invariantes de la concepción geométrica}

El límite se aplica a magnitudes geométricas, como áreas, volúmenes, superficies barridas, ángulos de rotación. En la época griega se considera como una aproximación de procesos geométricos infinitos, dada por la intuición geométrica o espacial y en el Renacimiento el límite se considera como una "aproximación finita", como que toma "una" de un número finito de cantidades que se aproximan al limite como la mejor aproximación, y no tanto como se desee. El infinito que es un concepto consustancial a la noción de límite se rechaza y evade en matemáticas, generándose el obstáculo "Horror al infinito" (Cantor) que impide ver el proceso de aproximación como una operación que llega a un resultado (Limite).

\section{Sistemas de representación de la concepción geométrica}

Aproximaciones de áreas mediante construcciones geométricas con regla y compás, basadas en iteraciones indefinidas. Suma infinita de magnitudes infinitamente pequeñas. Aproximaciones de volúmenes mediante cálculos numéricos. Fracciones decimales y la notación decimal (Stevin) 


\section{CONCEPCIÓN HEURÍSTICA DE APROXIMACIÓN CINÉTICA INFINITESIMAL NEWTON}

El limite es una cantidad (Cociente) a la cual una razón de cantidades en movimiento se aproxima continuamente, mas que cualquier diferencia dada y no puede alcanzarla o sobrepasarla antes que las cantidades hayan decrecido indefinidamente.

HEURÍSTICA: Métodos heurísticos de inducción incompleta, analogía, interpolación lo llevó a métodos algorítmicos y fórmulas universales.

APROXIMACIÓN CINÉTICA: Guiada por su conocimiento de la Física y de a variación continua sugerida por el movimiento.

VARIABLES: "Fluentes", magnitudes geométricas o físicas asociadas al movimiento, que varían con el tiempo. El tiempo es la variable independiente universal.

FUNCIÓN: Expresión analítica. El conjunto de los números reales no está formalizado.

Uno de los aportes más relevantes de Newton a la evolución de la noción de límite, fue darse cuenta que para calcular una razón de cambio instantánea (velocidad instantánea) era necesario considerar procesos infinitos entre dos valores suficientemente próximos. Su centro de interés fue la resolución de problemas de movimiento y variación que implican el cálculo de velocidades, distancias, aceleraciones. Problemas de Tangentes y cuadraturas. Rectificación de curvas, Máximos y mínimos y series infinitas.

La noción de límite transita implícitamente en sus métodos, específicamente cuando calcula la Fluxión: velocidad de cambio de la fluente (magnitud que fluye o varía con el tiempo). Pero en donde está más cerca de la idea de límite es un su método de "Primera y última Razón de cantidades nacientes o evanescentes" las que describe en su "Principia" así:

\subsection{Concepción de Leibniz (1 646-1 71 6)}

"Cocientes (o razones últimas) en las que las cantidades se anulan, no son estrictamente hablando, razones de cantidades últimas sino límites a donde se acercan las razones de esas cantidades, al decrecer sin límite, las cuales aunque pueden hacerse más próximas a sus limites que cualquier diferencia dada, no pueden ni sobrepasar/os ni alcanzarlos antes que las cantidades hayan decrecido indefinidamente"... Por razón última de cantidades evanescentes debe entenderse la razón de cantidades, no antes de que se anulen, no después, sino aquella con la que se anulan. (Kline, 1992, p. 482)

\section{Invariantes de la concepción de Newton}

El límite se aplica a "magnitudes variables" que involucran tanto magnitudes geométricas como físicas Se interpreta como aproximación infinitesimal cinética tanto 
como se desee, lo cual se traduce en una "aproximación infinita" equivalente a 'aproximar tanto como se quiera" o a 'la aproximación con cualquier error". Aunque la concepción de Newton no acepta explícitamente el infinito actual, su actitud hacia el infinito puede considerarse "actualista potencial" en cuanto considera el limite como el último término surgiendo en el tiempo y cuando todos están, allí está el límite (Sierpinska, 1987), porque encuentra resultados de procesos infinitos como las razones de magnitudes evanescentes.

\section{Representaciones}

Razón de cambio instantáneo (razón última). Pendiente de tangentes a curvas. Sumas de series infinitas, aproximaciones numéricas mediante procesos iterativos. Representaciones gráficas de curvas, algebraicas y numéricas.

\section{CONCEPCIÓN METAFÍSICA \\ ALGEBRAICA \\ INFINITESIMAL - LEIBNIZ}

Límite es un "ente último" tal, que existe una diferencia infinitesimal entre él y los valores que se le aproximan, tanto como se quiera.

METAFÍSICA: "Principio de Continuidad"

ALGEBRAICA: Predominio de simbología, fórmulas y algoritmos generales.

INFINITESIMAL: Considera 'el diferencial” como la diferencia infinitamente pequeña, a veces ínasignable o cuantitativamente cero.

VARIABLES: Recorriendo una sucesión de valores infinitamente próximos.

DEFINICIÓN DE FUNCIÓN y DE NÚMERO REAL: Están ausentes. La función es una expresión analítica y la diferencia entre 1 y $0.99999 . .$. es el segmento que falta por recorrer más que un número.

Igual que Newton, estuvo interesado en resolver problemas de movimiento y variación, pero mientras que a Newton le interesaba la descripción científica de la generación de magnitudes, a Leibniz le preocupaba la explicación metafísica de tal generación (Boyer, 1959, p, 306). Sus métodos estuvieron más orientados por el trabajo aritmético de sucesiones de sumas y diferencias, reconoce la potencia del álgebra y su simbolismo para el cálculo infinitesimal es el aceptado por la comunidad matemática. La idea de límite de Leibniz estuvo más orientada por su preocupación filosófica y la basé en su principio de continuidad:

"En cualquier supuesta transición que acaba en un término, es válido elaborar un razonamiento en el que el término final quede incluido" (Kline, 1992, p. 510)

\section{Invariantes de la concepción de Leibniz}

El límite se aplica a "cantidades variables", relativas a valores numéricos, las que considera con una visión estática en el sentido de valores fijos pero indeterminados. El 
modelo de límite es "infinitesimalista" por considerar diferencias infinitamente pequeñas. Su actitud hacia el infinito también es "actualista potencial", por cuanto admite que después de un tiempo la sucesión infinita será completada y sus términos estarán disponibles. (Sierpinska, 1987).

\section{Sistemas de representación}

Razones entre magnitudes, triángulo diferencial, simbología específica de derivada e integral. Series infinitas y representaciones geométricas, algebraicas y aritméticas. Representaciones gráficas de curvas.

\subsection{Concepción algebraica}

La noción de límite transita implícitamente en los algoritmos y las fórmulas algebraicas. Esta concepción está determinada por los trabajos de los matemáticos que usaron el álgebra como herramienta para obtener resultados a problemas que involucraban la noción de límite.

\section{CONCEPCIÓN ALGEBRAICA \\ FINITISTA - FERMAT \\ (Comienzos del S. XVII)}

El paso al limite es una operación matemática que consiste en aplicar números a las variables y omitir valores despreciables respecto de otros. (Sierpinska, 1987).

ALGEBRAICA: El incremento E tiene cualquier valor fijo. La "adigualdad" es un proceso algebraico, por equivalencias. Se busca una fórmula mágica para resolver los problemas del mismo tipo.

FINITISTA: E es una variable finita como en las ecuaciones de Vietá, en el sentido de un valor fijo pero indeterminado.

FUNCIÓN: Expresión analítica. 
Es el primer matemático que tiene la idea de tomar un incremento diferencial en la que Ilamamos variable independiente y analizar el comportamiento de la variable dependiente en su método de "Adigualdad" para hallar máximos y mínimos de curvas polinómicas y que luego extiende a las tangentes. Este método no implica ningún concepto de límite sino que es algebraico.

\section{CONCEPCIÓN ALGORÍTMICA \\ ALGEBRAICA - EULER (1701-1783)}

El paso al limite queda disimulado cuando considera apropiado tomar las cantidades infinitamente pequeñas como ceros (finitas) pero sus razones pueden ser finitas (Limite).

ALGEBRAICA: Ios diferenciales son cantidades finitas.

ALGORÍTMICA: Privilegia las fórmulas y los algoritmos más que las demostraciones y poco se preocupa por la búsqueda de los Fundamentos del Cálculo Infinitesimal

FUNCIÓN: Expresión analítica.

Euler y los Bernoulli tratando de resolver problemas de Geodesia, Física, Mecánica, navegación, calendarios, movimiento de proyectiles, diseño de lentes, que exigen conocimiento cuantitativo desarrollaron y ampliaron el cálculo de Leibniz y generaron nuevas ramas de la matemática. Euler usa los diferenciales de Leibniz que los considera cero cuando es apropiado. El argumenta que $d y / d x$, que para él era 0/0 podía ser un número bien definido, usando propiedades del álgebra finita. A Euler le interesa más que todo los resultados y poco los fundamentos de los métodos, tanto que dice: "Para qué probar lo más evidente con lo que es menos evidente" (Boyer, 1959)

\section{CONCEPCIÓN RIGUROSA \\ FINITISTA - LAGRANGE (1736 - 1813)}

No se admite la idea de límite, ni transita implícitamente en los métodos algebraicos.

FUNCION: Expresión analítica

Es el primer matemático que se interesa por buscar los fundamentos del Cálculo Infinitesimal de Newton Y Leibniz. Pero como se ve influenciado por el "Horror al infinito", para deshacerse del infinito y los infinitesimales, busca la fundamentación del Cálculo en el Algebra en donde usa como herramienta las Series de Taylor. Su propuesta "Teoría Analítica de funciones sin límites" contribuye a independizar el Análisis de lo geométrico y lo mecánico. 


\section{Invariantes de la concepción algebraica}

El límite se aplica a "cantidades variables en expresiones analíticas $(y=f(x))$ " Las cantidades variables hacen referencia a valores de magnitudes físicas o geométricas, en el sentido de valores fijos pero indeterminados. Aquí hallar el límite consiste en aplicar algoritmos y artificios algebraicos. Predomina la actitud finitista ya que el infinito se considera como un número muy grande o se rechaza.

\section{Sistemas de representación}

Incrementos fijos de las variables. Representaciones gráficas y algebraicas de curvas. Se introduce el símbolo $\mathrm{f}(\mathrm{x})$ para función y esta se concibe como una expresión analítica $(y=f(x))$

\subsection{Concepción aritmética}

Se consideran tres fases:

S. XVII Transición de la concepción geométrica a la Aritmética cuyo representante es Walls.

S. XVIII Siguiendo la línea de la búsqueda de los fundamentos del Cálculo infinitesimal, D'Alembert es el primero en proponer la "Teoría de limites" como un alternativa de solución.

S. XIX Cauchy inicia el camino hacia la formalización del concepto.

\section{CONCEPCIÓN ARITMETICA HEURÍSTICA INFINITISTA-WALLIS (1661-1703)}

El paso a límite no es una operación matemática sino un método heurístico que conduce a hallazgos gracias a razonamientos basados en inducción incompleta.

(Sierpinska, 1987, p. 372)

ARITMÉTICA: Métodos Geométricos $\Rightarrow$ Métodos Aritméticos.

HEURISTICA: Inducción incompleta, interpelación. Su lema es: "Primero inventar y luego probar".

INFINITISTA: Se ibera del temor al infinito heredado de los griegos. Trabaja con el infinito en series infinitas e induce el símbolo $\infty$ 


\section{CONCEPCIÓN GEOMÉTRICA-ARITMETICA DINÁMICA - DALEMBERT \\ $(1717-1783)$}

El límite de una cantidad variable es visto como una cantidad fija a la cual se acerca o aproxima tanto como se quiera (con un error menor que cualquier cantidad dada)

GEOMÉTRICA - ARIMÉTICA: Magnitud física ó geométrica $\Rightarrow$ Cantidad Variable

D'Alembert es el primero que propone explícitamente como una solución al problema de los fundamentos del Cálculo infinitesimal "La Teoría de Límites" siguiendo la tradición de cantidades variables. Así con base en las definiciones de Jurin (1734) y Robins (1735) que interpretan la noción de límite de Newton, publica en la Enciclopedia de Diderot la siguiente definición:

"Se dice que una cantidad es el límite de otra cantidad cuando la segunda puede aproximarse a la primera tan cerca como una cantidad dada, tan pequeña como se pueda suponer, sin que la cantidad que se acerca pueda sobrepasar la cantidad a la que se acerca; de suerte que la diferencia de una semejante cantidad a su límite es absolutamente inasignable" (D’Alembert, 1765 en Enciclopedie, Artículo "Límite" citada por Sierpinska, 1985, p.49)

\section{CONCEPCIÓN NUMÉRICA DINÁMICA INFINITESIMAL - CAUCHY (11789-1 857)}

Límite como valor fijo al cual se aproximan indefinidamente valores numéricos de una variable en tanto como se desee.

Cantidad variable $\Rightarrow$ Variable Numérica dinámica.

FUNCION: como dependencia entre variables. Es el "Conjunto de los valores" que toma la variable dependiente.

A él se le atribuye el honor de ser el primero en institucionalizar el concepto de límite como "objeto matemático" ya que el concepto de límite antes se usaba como una "noción instrumental" en los procesos de aproximación. La principal situación que lleva a Cauchy a la búsqueda del rigor es su compromiso de Enseñanza del Curso de Análisis, en el cual se propone alejarse de la manipulación de fórmulas y figuras geométricas. Empieza por definir variable, límite e infinitesimal. Así, define límite en su "Cours de Análise", tomando como referencia la definición de D’Alembert:

"Cuando los sucesivos valores que toma una variable se aproximan indo finida-mente a un valor filo, de manera que termina por diferir de él en tanto como queramos, este último valor se llama límite de todos los demás" (Cauchy citado por Boyer, 1992, p. 647) 


\section{Invariantes de la concepción aritmética}

Se habla de limite de una "variable numérica dinámica". Algunos autores (Kline, 1992) interpretan la definición de Cauchy como Límite de una sucesión por aplicarla a los sucesivos valores que toma una variable y otros como límite de una función refiriéndose solo a la variable dependiente. El límite se ve como una aproximación tan precisa como se desee entre valores numéricos (aproximación infinita), y el uso de las expresiones dinámicas "aproximarse indefinidamente" y "diferir tanto como se desee", empiezan a encapsular los procesos infinitos implicados, generando los modelos dinámicos de limito como frontera a veces alcanzable y otras veces inalcanzable.

\section{Sistemas de representación}

Simbología aritmética y algebraica, usadas en procesos infinitos (sumas, productos, cocientes, expansiones decimales). Definiciones verbales de límite. Notación de límite como operación y Simbología de operaciones y relaciones de entre números reales.

\subsection{Concepción analítica - Weierstrass (1815- 1897)}

\section{CONCEPCIÓN ARITMÉTICA ANALÍTICA ESTÁTICA - WEIERSTRASS}

El limite es un concepto riguroso y estático en términos de números reales, sus relaciones y operaciones y sirve para demostrar teoremas generales de clases de funciones.

ARITMÉTICA: "Aritmetización del Análisis":

fundamentar las matemáticas en $\mathrm{R}$

ANALITICAs El limite se refiere a una función, y sobre este se estructura todo el Análisis.

RIGUROSA: Definición formal y precisa.

ESTÁTICA: La variable continua se define en forma estática.

Variable Dinámica $\Rightarrow$ Variable estática

(Recorriendo un conjunto (Representando uno cualquiera de los valores)

El padre del rigor y la formalización de las matemáticas, Weierstrass, se encuentra en mitad del siglo XIX , cuando la Matemática sufre la crisis de fundamentos. La base sobre la cual estaba construida la matemática, la Geometría, se "desestabiliza", con la aparición de las geometrías no Euclideas. Entonces, los fundamentos del Cálculo infinitesimal no se podían buscar en la geometría y es Weierstrass quién inicia el programa de "aritmetización del Análisis", que consistía en fundamentarlo en el concepto de "número real".

Para llegar a la definición de límite, se requirió precisar 'Número real", para lo cual se necesita el concepto de "conjunto infinito" que incorpora el "infinito actual"; tarea que emprenden simultáneamente Cantor, Dedekind, Weierstrass y Heme. 
Weierstrass resuelve la cuestión de la existencia del límite de una sucesión convergente haciendo la sucesión en sí misma el límite o el número "(Boyer, 1959, p. 254). Esta apreciación lleva implícita la consideración del infinito actual que le permite ver el "límite como el objeto creado por el proceso infinito terminado". Para él la sucesión 0.3, $0.33,0.333,0.3333, \ldots=1 / 3$

Y así presenta la siguiente definición de límite:

" $L$ es el limite de una función $f(x)$ para $x=x_{0}$, si, dado arbitrariamente cualquier número pequeño $\varepsilon$ puede ser encontrado un número $\delta$ tal que para todo valor de $x$ difiriendo de $x_{0}$ por menos que $\delta$, el valor de $f(x)$ diferirá de $L$ por menos el valor de $\varepsilon$ " (Weierstrass, citado por Boyer, 1959, p. 287)

Esta definición la hizo más formal Heme, con la introducción de los cuantificadores y la definición de función de Dirichlet y Riemann como correspondencia arbitraria en 1872:

'Si dado cualquier $\varepsilon$ existe $\eta_{0}$ tal que para $0<\eta<\eta_{0}$, la diferencia $f\left(x_{0}, \pm \eta\right)-L$ es menor en valor absoluto que $\varepsilon$, entonces se dice que $L$ es el límite de $f(x)$ para $x=x_{0}$ (Heine - Weierstrass, en Boyer, 1992, p. 696)

Hoy la $\eta$ (de Weierstrass), se ha reemplazado casi universalmente por $\delta$ y la definición se conoce así:

$L$ es el límite de $f$ en $x=p$ si $A \varepsilon>0, Э \delta>0$ tal que $0<|x-p|<\delta \Rightarrow|f(x)-L|<\varepsilon$

En esta definición no se da la imagen de cantidades en movimiento, o cantidades variables que se aproximan a un valor o de puntos moviéndose en una curva.

\section{Invariantes de la concepción analítica}

El límite se aplica a "funciones", pero las funciones vistas como correspondencia arbitraria entre números, donde prima la unicidad. Esta concepción refleja una visión estática de límite. Se ocultan los proceso infinitos y se destierran las ideas de variabilidad continua, de aproximación e infinitésimos constantes o variables.

\section{Sistemas de representación}

A través de los reales, de funciones reales. Definición simbólica $\varepsilon-\delta$

\section{PROBLEMAS CONCEPTUALES PARA LA EVOLUCIÓN DE LA NOCIÓN DE LÍMITE}

La noción de límite evoluciona tratando de dar respuesta a los siguientes problemas planteados al interior de las matemáticas:

\section{P1 - La relación entre número (lo discreto) y magnitud (lo continuo)}

Surge de las paradojas de Zenón y el descubrimiento de los inconmensurables, separando lo geométrico de lo numérico y a pesar de los aportes para solucionarlo como los de: Stevin (s. XVI) con su planteamiento sobre el concepto de "número", Descartes y Fermat con la geometría Analítica, Newton y Leibniz que asocian números a magnitudes y 
viceversa y Cauchy que hace transferencia del continuo geométrico al numérico, solo es resuelto definitivamente por Dedekind (1831 - 1916) cuando define "continuo numérico" y "recta analítica" en donde coexisten los discreto y lo continuo y se es consciente que tanto los número reales como los puntos de la recta son "construcciones intelectuales" y no dependen de la intuición física o espacial.

\section{P2 - Naturaleza de los elementos infinitesimales}

Nace con las primeras versiones de los métodos infinitesimales y se moviliza en los métodos de Newton y Leibniz, los cuales son cuestionados y criticados Por ej. por Berkeley). Se refiere especialmente a la naturaleza de los "incrementos fijos" o "incrementos variables" o "elementos infinitesimales" y se soluciona con Cauchy cuando define "infinitésimo" y Límite" en el marco numérico.

\section{P3 - Búsqueda del rigor en el álgebra}

Es la tendencia a buscar el rigor de los métodos del cálculo en el álgebra, para huir del infinito y los infinitésimos, especialmente por Lagrange (S. XIX)

\section{P4 - Razonamiento circular en las definiciones de límite y de número irracional}

Surge de la definición de límite de Cauchy en la cual asume al existencia de los números reales, para definir límite y luego define número irracional (real) como límite de sucesiones de racionales.

\section{OBSTÁCULOS EPISTEMOLÓGICOS}

\section{Oi - "Horror al infinito"}

Surge del problema entre lo discreto y lo continuo en la Matemática griega. Los griegos desterraron el concepto de infinito en Matemáticas y todo los que tuviera que ver con el infinito, y este fue el motivo para idear el método de demostración indirecta o por reducción al absurdo. Pero más que considerar el temor al infinito heredado de los griegos, el "Horror al infinito", expresión acuñada por Cantor, se refiere a la no aceptación del "infinito actual" en matemáticas, es decir la no aceptación de un proceso infinito completado o terminado.

Aunque se empieza a desmitificar el infinito en la edad media con los trabajos de Oresme y en el renacimiento con las reflexiones de Galileo sobre el infinito actual, solo hasta los trabajos de Wallis se manipula el infinito libremente, luego en le Cálculo infinitesimal de Newton y Leibniz y el Análisis Infinitesimal de Euler, las series infinitas se convierten en herramientas potentes para el desarrollo de las matemáticas, pero todos ellos incluso Cauchy se vieron afectados por este obstáculo, y solo es completamente superado con la teoría de conjuntos de Cantor y la aceptación de 'infinito actual "en matemáticas el cual permite definir limite en el contexto numérico.

\section{Og v n - Separación de lo geométrico y lo numérico}

Ligado a los problemas de la relación entre lo discreto y lo continuo y el descubrimiento de los inconmensurables. 


\section{Og — Obstáculo geométrico — Paradigma euclídeo}

Básicamente dificultó hacer transferir la idea de límite de lo geométrico a lo numérico. El modelo de razonamiento geométrico Euclídeo era el perfecto y a pesar de que se habían hecho trabajos para superarlo como los de Wallis, Newton, Leibniz, Euler, solo se logra superar totalmente con el descubrimiento de las Geometrías no Euclídeas y la Aritmetización del Análisis.

\section{Of a i - Transferencia de lo finito a lo infinito}

Se inicia desde al edad media, cuando Oresme y Calculator incorporaron el infinito en los trabajos de sumas infinitas y luego se acentúa en los trabajos sobre series infinitas de Wallis [Ej. ( $\mathrm{a} /(\mathrm{)})$ ( = a, Newton, Leibniz, Bernoulli, Euler ya encuentran inconsistencias con algunas series infinitas como la serie de Grandi: $1-1+1-1+1 \ldots$ y solo es librado por Cauchy quien reconoce que los procesos infinitos requieren tratamientos diferentes a los finitos y que el cálculo necesita razonamientos y tratamientos diferentes a los algebraicos y geométricos.

Oc - Principio de continuidad de Leibniz - (transferir una propiedad de una sucesión convergente a su límite)

Por ejemplo se manifiesta cuando se cree que el límite de $0.9,0.99,0.999$, tiene la misma propiedad de sus términos y por consiguiente debe ser menor que 1 . Este se extiende en los trabajos de Bernoulli, Euler y D'Alembert.

\section{Of(x) - Obstáculo relativo a funciones}

Se genera de la concepción que tiene Euler de Función como expresión analítica y = $f(x)$, concepción que se puede extender a Newton y Leibniz en donde no se mira la naturaleza de los $x$ y los y. Se supera cuando se precisa el concepto de función real de variable real, función continua y esto se logra simultáneamente con la institucionalización del concepto de límite.

\section{OL - El límite se alcanza o no}

Surge por el uso de expresiones dinámicas en las primeras definiciones de limito de Jurín, Robins, D’Alembert, Lhuilier y Cauchy por la influencia del infinito potencial. Es superado cuando Weierstrass, haciendo uso del infinito actual, considera el "limite" como el objeto creado por el "proceso infinito". Por ejemplo establecer que 0.3, 0.33, 0.333, $0.3333=1 / 3$

\section{OS - Obstáculo de la simbología}

Es el producido por el uso de la simbología propia de la matemáticas y la inserción de cuantificadores de la lógica. La simbología utilizada denota semejanzas con el álgebra, esconde las diferencias llevando a una pérdida de significación (Sierpinska, 1985, p. 55). En ella se encapsulan los procesos infinitos y situaciones que le dan sentido a la noción de límite y oculta su complejidad. 


\section{DIFICULTADES}

Ligadas a los obstáculos aparecen dificultades para la evolución de la noción de límite; son menos resistentes que los obstáculos epistemológicos pero los refuerzan. Las dificultades identificadas que nacen en al antigüedad griega son: la influencia de la intuición geométrica, el excesivo rigor en los métodos y falta de hallar resultados directos, en la edad media y renacimiento surgen el carácter empírico y heurístico de las matemáticas que impide la explicación de los resultados, luego surgen las dificultades asociadas al álgebra como influencia de la posición finitista del álgebra de Vietá (s. XVII) que lleva a ver el cálculo como una extensión del álgebra (s. XVIII). Por otro lado surgen las dificultades generadas por el uso de expresiones dinámicas (finales del siglo XVIII y comienzos del s. XIX) y en las cuales se detecta la falta de la noción clara de conjunto infinito y la omisión de una definición clara de número (s. XIX), para poder llegar a la definición forma! y simbólica de límite, la cual también ha generado dificultades didácticas. A continuación se muestra tal transición:

\begin{tabular}{|c|c|c|c|c|c|}
\hline $\begin{array}{l}\text { Carácter } \\
\text { Riguroso }\end{array}$ & $\begin{array}{l}\text { Carácter } \\
\text { Empírico }\end{array}$ & $\begin{array}{l}\text { Carácter } \\
\text { Heurístico }\end{array}$ & $\begin{array}{l}\text { Predominio } \\
\text { de fórmulas }\end{array}$ & $\begin{array}{l}\text { Uso de expres. } \\
\text { dinámicas }\end{array}$ & $\begin{array}{l}\text { Carácter } \\
\text { formal }\end{array}$ \\
\hline $\begin{array}{l}\text { S.VIA.C. } \\
\text { a S.II }\end{array}$ & $\begin{array}{l}\text { S.XIV } \\
\text { a S.XV }\end{array}$ & $\begin{array}{l}\text { S.XVI } \\
\text { a SXVII }\end{array}$ & S.XVIII & $\begin{array}{l}\text { S.XVIIIa } \\
\text { a comienzos XIX }\end{array}$ & S.XIX \\
\hline
\end{tabular}

A finales del siglo XIX y durante el siglo XX, se ha objetado la eliminación de los infinitesimales, procesos infinitos y las ideas de aproximación y variabilidad en la definición de Weierstrass. Al respecto comenta Cornu:

"A pesar de los intentos de desvanecer los infinitesimales del Análisis moderno, estos continúan vivos en las mentes y comunicaciones de los matemáticos profesionales. El regreso de los infinitesimales basados en el trabajo de Robinson (1966), la construcción de un sistema hiperreal que contuviera números reales e infinitesimales, reabrió el debate, el cual continua siendo muy confrontado" (Cornu, 1991, p. 162, 163).

\section{PROCESO DE INSTITUCIONALIZACIÓN DEL CONCEPTO DE LÍMITE}

Es notoria la lentitud del proceso de institucionalización del concepto de limite, proceso que duró aproximadamente 25 siglos. En su primer estatus (protomatemático), como idea que se moviliza implícitamente en los razonamientos, se extendió desde el siglo $\mathrm{VI}$ a. O. hasta el siglo II porque los griegos huyeron a todo aquello que no tuviera interpretación lógica y no fuera evidente por la experiencia física, por lo tanto desterraron los conceptos ligados a la noción de límite como: infinito, variación, continuidad, infinitesimal.

En el siglo XIV, la iniciación del estudio del cambio y la introducción del infinito en matemáticas, abre el camino para que los matemáticos de los siglos XVI y XVII ideen 
métodos heurísticos en los cuales transita la noción de límite como instrumento útil (estatus paramatemático) para resolver problemas de cálculo diferencial e integral, y luego con un enfoque algebraico se genera la creación de nuevas ramas de la matemática en el siglo XVIII, pero aún no está definida ni fundamentada la noción de límite.

Solo inicia su fase hacia el estatus matemático con las primeras definiciones a finales del siglo XVIII, luego se toma conciencia de su función en la estructura del campo del cálculo, se define en forma rigurosa para ser aceptada por la comunidad matemática y así convertirse en objeto de estudio de las Matemáticas (s. XIX)

Este análisis se complementa con la Teoría de Sfard (1991), según la cual conceptos matemáticos como el de límite, se desarrolla primero en un gran proceso de trabajo procedimental u operacional (s. VI a. O. - SXVIII), antes de llegar a encapsularse en una definición o una teoría, que sería la fase estructural o formal del concepto (s. XIX)

\section{CONSIDERACIONES FINALES}

El estudio epistemológico de la noción de límite permite reconocer que su génesis y proceso de institucionalización fue bastante difícil y controvertido y que la forma en que a comunidad matemática lo manipuló en cada época da origen a las diferentes facetas que generan la complejidad del concepto. Además se advierte que el conocimiento matemático no es el resultado de un proceso continuo y progresivo; por el contrario, la evolución de la noción de límite requiere de momentos de ruptura con conocimientos anteriores, se aprecian avances, retrocesos, desvíos, retornos, dependiendo de las dificultades por vencer y obstáculos por superar en cada época.

Las ideas que conforman las concepciones generadas durante el proceso de evolución como: límite de procesos infinitos, ideas de aproximación finita e infinita, límite de magnitudes variables, cantidades infinitamente pequeñas e infinitamente grandes, quedan ocultas en la definición formal, lo cual lleva a formular numerosos interrogantes acerca del tratamiento didáctico del concepto de límite. Por ejemplo: Es posible acercar a la comprensión del concepto a partir de la definición formal $\varepsilon-\delta$ ? La comprensión del concepto se puede lograr mediante la secuencialización y temporalización tradicional de los contenidos? Tiene sentido hacer un estudio independiente de límites, como introducción al cálculo, con tratamientos formales y rigurosos sin ubicarlos en algún escenario que involucre la noción? Los acercamientos al cálculo por medio de la revisión de algoritmos y artificios algebraicos son apropiados para inducir al pensamiento variacional del cálculo?

Pero, antes de reflexionar sobre las posibles respuestas a tales interrogantes, se hace necesario hacer el estudio de las concepciones o imágenes que los estudiantes se están formando sobre de la noción de límite, para determinar que tanto se acercan o alejan del concepto y en un futuro poder presentar alguna propuesta didáctica.

\section{BIBLIOGRAFÍA}

Artigue, M. (1998). Enseñanza y aprendizaje del análisis elemental: ¿Qué se puede aprender de las investigaciones didácticas y los cambios curriculares? Revista Latinoamericana de Investigación en Matemática Educativa. $\mathrm{N}^{0} 1$, p. 40-55. 
Bachelard, G. (1993). La formación del espíritu científico. Siglo XXI editores, S. A. México.

Boyer, C.B. (1959). The History of the Calculus and its Conceptual Development. Dover Publications, Inc. New York.

Chevallard, Y. (1991) La transposición didáctica: del saber sabio al saber enseñado. Argentina: Copyright Aique Grupo editor S.A.

Cornu, B. (1991). Limits. En: Advanced Mathematical Thinking. Netherlands: Kluwer Academic Publishers. P. 153-165.

Delgado, G. C. (1998). Estudio Microgenético de esquemas conceptuales asociados a definiciones de limite y continuidad en universitarios de primer curso. Tesis Doctoral. Universitat Autónoma de Barcelona.

El Bouazzaoui, H. (1988). Conceptions des éleves et des prof esseu rs a propos de la notion de continuité dune function. Thése Ph.D. Université Laval.

García, G.; Serrano, O.; Días, H. (1999). Una aproximación epistemológica, didáctica y cognitiva al cálculo. En la Revista de la Facultad de Ciencia y Tecnología No. 5, p. 5158. Universidad Pedagógica Nacional, Santafé de Bogotá.

González, U. P. (1992). Las raíces del Cálculo Infinitesimal. Alianza Editorial, S. A. Madrid.

Kline, M. (1992). El pensamiento matemático de la antigüedad a nuestros días, I, Alianza, Editorial SA. Madrid.

Ruiz Higueras, L. (1993). Concepciones de los alumnos de secundaria sobre la noción de función: Análisis epistemológico y didáctico. Granada, Tesis Doctoral. Universidad de Granada.

Sánchez, O. y Contreras, A. (1998). Análisis de manuales a través del tratamiento didáctico dado al concepto de límite de una función: una perspectiva desde la noción de obstáculo. En Enseñanza de las Matemáticas, V. 16, N0 1. p. 73-84.

Sfard, A. (1991). Qn the dual Nature of Mathematical Conceptions: Reflections en Processes and Objects as Difterent sSides of the Same Coin. Educational Studies in Mathematics 22, pp. 1 - 36.

Sierpinska, A. (1987). Humanities students y epistemological obstacles related te limits. Educational Studies in Mathematics 18, p 371 -397.

Tall, D. y Vinner, S. (1981). Concept Image y Concept Definition in Mathematics with particular reference te Limits and Continuity. Educational Studies in Mathematics 12, p. 151169. 